\title{
Envejecimiento saludable y deterioro cognitivo.
}

\author{
Healthy aging and cognitive impairment.
}

Sheila Castro-Suarez ${ }^{1, a ; 2, \text {; ; 3,b. }}$

Nuestro país ha experimentado en las últimas décadas un cambio demográfico significativo. En los años cincuenta nuestra población estaba constituida por niños, los adultos mayores (personas con 60 años a más) representaban solo el 5,7\% de la población; sin embargo, para el año 2018 representan el 10,4\% (1). De igual forma, las poblaciones en el mundo están envejeciendo; pero, la evidencia de que el aumento de la longevidad está acompañada por un período prolongado de buena salud es escasa (2); incluso podría no ser sostenible y enmascarar desigualdades dentro del mismo país; debido a que una persona pueda tener más de una enfermedad al mismo tiempo. Esta transición demográfica debería acompañarse de un envejecimiento saludable; con calidad de vida, menor morbilidad, y pocos años de discapacidad (3).

La Organización Mundial de la Salud redefine el envejecimiento saludable como "El proceso de desarrollo y mantenimiento de la capacidad funcional que permite el bienestar en la edad avanzada"; esta definición se enfoca en la capacidad funcional, que permite a la persona satisfacer sus necesidades básicas, aprender, tomar decisiones, mantenerse activo, construir relaciones y contribuir a la sociedad. Resulta de la interacción de la capacidad intrínseca del individuo y las características ambientales relevantes. La capacidad intrínseca de un individuo está en relación con las capacidades físicas y mentales; que tienen que ver con la habilidad para caminar, ver, oír, pensar, recordar, etc. y está afectada por factores como la presencia de enfermedades y cambios relacionados con la edad. Mientras que las características ambientales incluyen el hogar, la comunidad y la sociedad en general; las personas y sus relaciones, actitudes y valores, las políticas sociales y de salud. Ser capaz de vivir en entornos que apoyan y mantienen su capacidad intrínseca y capacidad funcional es clave para un envejecimiento saludable $(2,4)$.

Personas mayores de 65 años experimentan algunos cambios en las capacidades cognitivas. Algunas de ellas son consideradas como parte normal del proceso de envejecimiento, como los cambios leves relacionados con la edad que pueden afectar la memoria verbal y visual, la capacidad para nombrar objetos de uso infrecuente, las habilidades visuoespaciales y un leve compromiso en la concentración o la atención; estos cambios podrían estar presentes en el contexto de un envejecimiento saludable. Si estas dificultades en las capacidades cognitivas son más severas e incluyen otros dominios cognitivos, es posible que la persona esté dentro de un cuadro de deterioro cognitivo leve o demencia; y tendrá el médico la difícil tarea de diferenciarla, debido a que los síntomas varían de persona a persona (4).

La demencia es la pérdida progresiva de las capacidades cognitivas que compromete la funcionalidad y por ende la autonomía de la persona; cuyo factor de riesgo más importante es la edad, este factor de riesgo quedó demostrado en nuestro medio en un estudio realizado en el Cercado de Lima donde se encontró una prevalencia de 2,40\% en el grupo de edad de 65 a 69 años, esta se incrementó a 33,07\% (IC 95\%: 29,98-36,20) en el grupo etario de 90 a 94 años (5). Por ello, debemos promover estrategias de envejecimiento saludable a través de hábitos de vida que fomenten la salud y el bienestar.

1 Revista de Neuro-Psiquiatría, Facultad de Medicina Alberto Hurtado, Universidad Peruana Cayetano Heredia. Lima, Perú.

2 Centro de Investigación Básica en Demencias y Enfermedades Desmielinizantes del Sistema Nervioso, Instituto Nacional de Ciencias Neurológicas. Lima, Perú.

3 Instituto Peruano de Neurociencias. Lima, Perú

a Editora Asociada; ${ }^{\mathrm{b}}$ Médico-Neuróloga. 
Diversas publicaciones sugieren que la combinación de una buena nutrición, actividad física y actividades mentales y sociales pueden proporcionar beneficios. El año 2016, se publicó un estudio en población europea, que incluyó en su análisis 5139 varones y 5909 mujeres de 6 países europeos. Evaluó el envejecimiento saludable (la autopercepción de buen estado de salud, las habilidades funcionales y la percepción del sentido de la vida) basado en una encuesta y analizó predictores de envejecimiento saludable. Concluye que los estilos de vida que involucren actividad física vigorosa o moderada, el consumo de verduras y frutas, el consumo regular de comidas y el consumo adecuado de líquidos están relacionados positivamente con un envejecimiento saludable, y se deben incluir dentro de las políticas de salud pública (3). El ejercicio físico se ha asociado también con la mejora del estado de ánimo, movilidad, y disminución del riesgo de caídas (4).

Además, los elementos psicosociales relacionados con la participación social, la creación de redes y la satisfacción con la vida también son considerados beneficiosos para la salud (3). El construir redes de apoyo social para reducir el estrés, compartir el proceso de envejecimiento con otras personas que están experimentando los mismos cambios y vicisitudes de entrar en los últimos años de vida. Probar nuevas actividades puede ser una excelente manera de desafiar a las redes cerebrales y hacer nuevas conexiones (4).

Las personas mayores necesitan atenciones médicas de rutina, en sistemas de salud que proporcionen servicios integrados; servicios centrados en la persona, cercano a sus hogares y que brinden cuidados que ayuden a mantener su capacidad intrínseca por el mayor tiempo posible. Estos servicios son apropiados para todas las edades y debe ser la piedra angular de cobertura universal de salud (6). Frente al aumento de la longevidad en todo el mundo, se necesita con urgencia una respuesta de salud pública coherente que abarque múltiples sectores y partes interesadas, no solo enfocada en los adultos mayores si no en las diferentes etapas de la vida.

\section{REFERENCIAS BIBLIOGRÁFICAS}

1. Instituto Nacional de Estadística e Informática. Población Adulta Mayor.Lima: Instituto Nacional de Estadística e Informática; 2018. (Fecha de acceso 10 de diciembre del 2018) Disponible en: https:// www.inei.gob.pe/biblioteca-virtual/boletines/ninezy-adulto-mayor/2018/1/

2. Beard JR, Officer A, de Carvalho IA, Sadana R, Pot AM, Michel J-P, et al. The World report on ageing and health: a policy framework for healthy ageing. Lancet Lond Engl. 2016; 387(10033):2145-54. DOI: 10.1016/S0140-6736(15)00516-4

3. Sowa A, Tobiasz-Adamczyk B, Topór-Mądry R, Poscia A, la Milia DI. Predictors of healthy ageing: public health policy targets. BMC Health Serv Res.
2016;16 (Suppl 5): 289. DOI: 10.1186/s12913-0161520-5

4. Memory and Aging Center. Healthy Aging. California: Memory and Aging Center; 2018. (Fecha de acceso 10 de diciembre del 2018) Disponible en: https://memory.ucsf.edu/healthy-aging

5. Custodio N, García A, Montesinos R, Escobar J, Bendezú L. Prevalencia de demencia en una población urbana de Lima-Perú: estudio puerta a puerta. An Fac Med. 2008; 69(4):233-8. DOI: http:// dx.doi.org/10.15381/anales.v69i4.1110

6. Beard JR, Araujo de Carvalho I, Sumi Y, Officer A, Thiyagarajan JA. Healthy ageing: moving forward. Bull World Health Organ. 2017; 95(11): 730-730A. doi: 10.2471/BLT.17.203745 